\title{
AN OVERVIEW OF MULTIMODAL VIDEO REPRESENTATION FOR SEMANTIC ANALYSIS
}

\author{
Janko Ćalić ${ }^{\dagger}$, Neill Campbell ${ }^{\dagger}$, Stamatia Dasiopoulou ${ }^{\ddagger}$ and Yiannis Kompatsiaris ${ }^{\ddagger}$ \\ ${ }^{\dagger}$ Department of Computer Science \\ University of Bristol \\ Bristol BS8 1UB, UK \\ e-mail: \{janko, campbell\}@cs.bris.ac.uk \\ ¥ Informatics and Telematics Institute \\ 1st Km Thermi-Panorama Road \\ Thessaloniki, GR-57001 Greece \\ e-mail: \{dasiop,ikom\}@iti.gr
}

Keywords: video representation, multimodality, content-based indexing and retrieval, semantic gap

\begin{abstract}
This paper gives an overview of approaches to video representation targeting semantic analysis for content-based indexing and retrieval. It highlights the major achievements of the existing methodologies and sheds new light to the challenges that are still unsolved. The problem of adaptive representation of digital multimedia is critically assessed and some novel ideas are presented. In addition, the concept of video multimodality is reevaluated and redefined in order to introduce the modalities like editing technique. An extensive literature survey on the topics involved is given.
\end{abstract}

\section{Introduction}

In the field of multimedia content-based retrieval there has been a plethora of interesting research work presented recently that focuses on the problem of semantic gap between low-level information extracted from the media and the user's need to meaningfully interact with it on a higher level. However, the majority of ideas follow a paradigm of finding a direct mapping from low-level features to highlevel semantic concepts. Not only does this approach requires extremely complex and unstable computation and processing [4], but it appears to be unfeasible unless it targets a specific and contextually narrow domain $[31,5,17]$. Little has been done to design a system capable of creating appropriate representations of video media on various levels of complexity and thus improve adaptability and reliability of multimedia retrieval. As given in [3, 33], the multimedia database stands as a central point of the modern creativity and thus the challenge to effortlessly interact with the large digital media collections is our prime goal. In addition, much of the recent research attempts to utilise the multimodal character of the video media $[51,32,42]$ but fails to fully exploit the underpinning information from these closely intertwined modalities.
This paper is an attempt to shed new light on multimodal video representation and to give a critical survey of publications in this field. The everlasting question of the optimal representation of digital video media, targeting not only content-based retrieval but content analysis in general, is reassessed in order to identify the reasons behind such a persistent problem as the sematic gap. There have been numerous literature surveys on the various aspects to content-based video indexing and retrieval $[44,47,51]$. However, the problem of appropriate multimodal representation has been practically ignored, since the choice of low-level features in current retrieval systems tends to be independent of the content and its semantics.

Following a similar approach to the problem of video representation, the concept of video media multimodality is critically rethought outlining the outstanding approaches that challenge this problem. To support these claims, a survey of two common approaches to multimodal video representation, opposite in their character, is given i.e. data driven and concept driven generation of representation models.

The structure of the paper is as follows. The following section gives a brief chronological introduction to the field of content-based video indexing and retrieval. In Section 3 the problem of video representation is presented, while Section 4 brings a novel perspective to the concept of multimodality in digital video media. Data-driven approaches to representation is given in Section 5 and top-down algorithms are presented in Section 6. The final conclusions are given in Section 7 followed by the list of the referred publications.

\section{Content-based video retrieval}

The first generation of visual retrieval systems had the attributes of visual data extracted manually. Such an attribute-based representation entailed high level of image abstraction and modeled visual content at conceptual level. Representation schemes like relational models and object-oriented models were used. The search engines worked in the textual domain and used either traditional query languages like SQL or full text retrieval. Cost of 


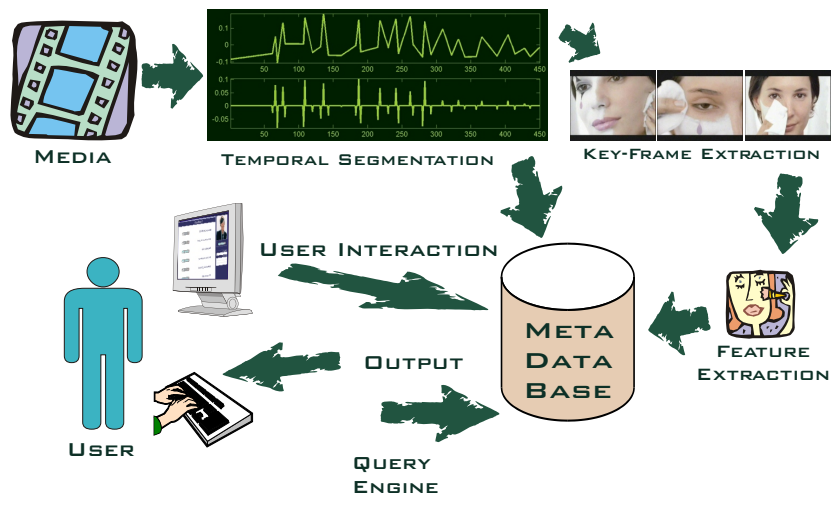

Figure 1: A typical second generation content-based retrieval system

annotation was typically very high and the whole process suffered from subjectivity of descriptions.

On the other side, the second-generation systems address perceptual features like colour, texture, shape, spatial relationships, etc. They concentrate on obtaining fully automated numeric descriptors from objective measurements of the visual content and support retrieval by content based on combinations of these features. The retrieval is based on similarity models that somehow replicate the way in which humans assess similarity between different objects. Unlike still images, video conveys informative messages through multiple planes of communication, i.e. audiovisual content is inherently multimodal. That involves the way in which the frames are linked together using various editing effects (cut, fades, dissolves, mattes, etc.) as well as the high level information embedded in a frame sequence (the characters, the story content, the story message). Text embedded in the video frames and the other sensory data like speech and sound can be employed to extract useful data. The system comprises automatic extraction of the video structure $[7,28,22,49,53]$ by detecting the edit effects that permit video composition, the extraction of the key-frames from the shots, and modelling perceptual content of these key-frames. In this way the problem of video retrieval by content has been reduced to the problem of retrieval by content of structured still images.

The video retrieval system depicted in the Figure 1 has the typical second generation structure with an additional user relevance feedback functionality. Initially, it segments video into its temporal units like shots or scenes and afterwards extracts a set of representative key-frames. Exploiting various image processing and computer vision techniques low-level feature descriptors are extracted and stored in a metadata database for later retrieval. User queries are transformed into the structurally equal feature descriptors and the search engine finds the most similar records from the metadata base. The relevance feedback unit monitors feedback given by user during the retrieval process and adapts feature descriptions in order to achieve more consistent results in terms of perceptual similarity.

Despite some effective results that have been reported in the literature, a key problem with second-generation retrieval systems remains bridging the semantic gap between the system and users. Virtually all the systems proposed so far use only low-level perceptively meaningful representations of pictorial data. However, similarity of perceptual properties is generally of little use in most practical cases of retrieval by content, if not combined with similarity of high-level information.

The third generation retrieval systems should be looking for higher level information from images, audio and video content. Who are the characters, their roles, the actions and their logical relations, as well as the emotional impact to the observer is the information that should be extract automatically, with no or minimal manual intervention, so as to support objective semantic-based retrieval. Much more that single images, retrieval of video is generally meaningful only if performed at high levels of representation. The user is much more concerned with the narrative structure of the video content than merely with perceptual elements of the video. Individual frames are not perceived as such. Instead user is affected by the rhythm of the edited sequence, composition of the scenes, etc. Therefore, the breaking point of the signification chain is the way system represents the content from the information embedded in the video sequence. We discuss this matter in the following section.

\section{Video representation}

In the context of semantic retrieval of video media, we address the problem of computational video representation, i.e. how to abstract the audio-visual experience of the user by means of computational models. This is clearly a difficult task that has to involve both appropriate computation and processing as well as the way in which a user experiences targeted media. However, this is not a common approach to video retrieval, where the focus is on the way information is extracted from the digital media, whether it makes sense to the user or not. As depicted in Figure 2, the information flow in a content-based video retrieval system has an important step between the set of automatically extracted low-level features and the user video representation.

The foundational work that has formulated the problem of computational video representation was presented by Davis [15, 14] and Davenport et al. [13]. In [14] multilayered, iconic annotations of video content called MediaStreams is developed as a visual language and a streambased representation of video data, with special attention to the issue of creating a global, reusable video archive. Being radically oriented towards a cinematic perspective of video representation, the work presented in [13] sets the scene to a novel approach to the content-based video analysis based upon a shot, an irreducible constituent of 


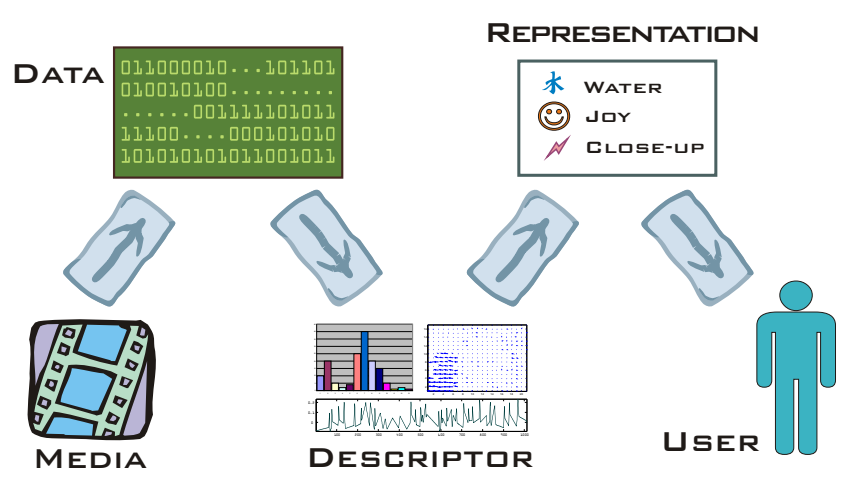

Figure 2: The representation step bridges computational information with the user

video sequences. But this was where research community stopped following this paradigm and got attracted to extraction and analysis of low-level features, ignoring the fact that these features would make little or no sense to the end user.

And it wasn't until the definition of Computational Media Aesthetics (CMA) in a number of publications by Dorai and Venkatesh $[19,18]$ that the user centered representation reemerged within the video retrieval community. The main idea behind CMA is to have a focus on domain distinctives, the elements of a given domain that shape its borders and define its essence (in film, for example, shot, scene, setting, composition, or protagonist), particularly the expressive techniques used by a domains content creators [1]. This is clearly a diametrically opposite point of view to the common perception that the video should be indexed by the terms for which automatic detectors can be realized [47]. Nevertheless, these two different approaches are bound to merge in order to achieve the goal of semantic retrieval of video media. Sections 5 and 6 outline work following these approaches, but let us first address the concept of multimodality in video media.

\section{Multimodality}

Seen from the generic system-centered perspective, multimodality is the capacity of the system to communicate with a user along different types of communication channels and to extract and convey meaning automatically [38]. However, the prevailing opinion is that the multimodality of video media is the capacity of an author to express a predefined semantic idea, by combining a layout with a specific content, using at least two information channels, where the channels can be either visual, auditory or textual [47]. Is it really true that these are the only communication channels by which the meaning of video is conveyed?

In recent publications challenging fundamental issues in content-based multimedia retrieval $[41,48,36]$ there has been an evident turn towards semiotic approach to the problem of the semantic gap. Semiotics is the study of signs and of the way meaning is transmitted and understood [43]. It has been applied widely in the analysis of film and video media, underlining the importance of communication through modes such as editing, narrative structure, visual composition (mise en scène), etc. The groundwork for the semiotic analysis in film theory was set by Eisenstein [20], Kuleshov [29] and later by Metz [34]. The Kuleshov experiment [23] indicated the importance and effectiveness of film editing by showing that juxtaposing two unrelated images could convey a separate meaning. Given the definition of multimodality [38] stated above, this implies that editing is a valid modality of video media. In this manner one could come up with more modalities that could be assigned to video media and ergo the widespread approach to multimodality as merging only visual, auditory and textual information seems to be hindering the development of the semantic analysis in content-based video retrieval.

However, a number of publications show a tendency towards a more sophisticated take on multimodality. The intensity and type of feeling or emotion, both referred to as affect, that are expected to arise in the user while watching a video clip have been computationally represented and modelled by Hanjalic and $\mathrm{Xu}$ [24]. In the recent publication by Dimitrova [16] an interesting proposition to exploiting concepts of short- and long-term memory in content analysis is presented. In addition, the influence between the multimedia modalities can be modelled through cross-modal association, as presented in the work by $\mathrm{Li}$ et al. [32].

Nevertheless, a clear definition of an infinite set of modalities is essential in order to make automatic classification feasible. On the other hand, the number of lowlevel features extracted from various modalities is limited. Therefore, a balanced interaction between feature driven bottom-up approaches and top-down algorithms could be a solution to a generic model for video representation.

\section{Data driven representations}

This approach is the standard way of extracting lowlevel features and deriving the corresponding representations without any prior knowledge of the related domain. Therefore, this is the hard-encoded way of representation driven by expert knowledge. A rough categorization of data-driven approaches in the literature yields two main classes $[52,2]$. The first class focuses mainly on signaldomain features, such as color histograms, shapes, textures, which characterize the low-level audiovisual content. The second class concerns annotation-based approaches which use free-text, attribute or keyword annotations to represent the content.

Many content-based indexing and retrieval systems have been proposed focusing mainly on the definition of suitable descriptors, the generation of appropriate metrics in the descriptors space and efficient addressing of 
the large workload and high complexity of the underlying image-processing algorithms. The existing systems fall broadly under four categories depending on the chosen content and indexing structures used: query by content, iconic query, SQL query and mixed queries. Query by content is based on images, tabular form, similarity retrieval (rough sketches) or by component features (shape, color, texture). The iconic query represents data with icons as its visual abstraction and specifies a query by the selection of appropriate icons. SQL queries are based on keywords, with the keywords being conjoined with the relationship (AND, OR) between them, thus forming compound strings. The mixed queries can be specified by text as well as icons. For a larger overview of relevant approaches a number of extensive surveys are available $[44,47,51]$.

Query By Image Content (QBIC) [21], currently in use by the Hermitage Museum for its online gallery, is an image retrieval system developed by IBM. It was one of the first systems to perform image retrieval by considering the visual content of images rather than textual annotations. QBIC supports queries based on example images, user-constructed sketches, and selected colors and texture patterns. In its most recent version, it allows text-based keyword search to be combined with content-based similarity search. It is commercially available as a component of the IBM DB2 Database System.

WebSeek [46] is a video and image cataloguing and retrieval system for the world-wide web developed at Columbia University. It automatically collects imaging data from the web and semi-automatically populates its database using an extendible subject taxonomy. Text and simple content based features (color histograms) are used to index the data to facilitate an iterative and interactive query method based on a Java and HTML search engine. VisualSeek [45] was also produced by Columbia University as an extension to WebSeek. It provides distinctly different functionality. Firstly it has not been specifically intended as a web-image search engine. Secondly it segments images to enable local and spatial queries. Segmentation uses the back-projection of binary color sets. This technique is used not only for the extraction of color regions but also for their representation.

The MUVIS [26] system has been initially created as a Web-based application during the late 90s to provide indexing and retrieval framework for large image databases using visual and semantic features such as color, texture and shape. During recent years, a new framework, which aims to bring a unified and global approach on indexing, browsing and querying of various digital multimedia types such as audio/video clips and digital images, has been developed, providing tools for real-time audio and video capturing, encoding by last generation codecs such as MPEG-4, H.263+, MP3 and AAC. A novel retrieval technique the so-called Progressive Query that provides faster and instantaneous periodic query results along with the ongoing query process. Furthermore MUVIS provides video summarization techniques developed over automatic, semi-automatic and supervised scene frame extraction from the shot-frames.

The framework developed within the SCHEMA [35] project considers the design and implementation of a reference system for content-based information retrieval. The proposed system employs the MPEG-7 XM (MPEG-7 eXperimentation Model) along with extensions developed specifically for the system to improve functionality and efficiency. In addition, the system supports high level descriptors and content-based indexing and retrieval using other modalities (e.g. pre-existing keyword annotations, text generated via automatic speech recognition (ASR)).

Although useful for representation of video within a limited domain, such approaches lack the capability to adapt to different domains. Indeed, the richness of audiovisual content is difficult to describe with a few keywords and data-driven representations, while content perception itself is a subjective and task-dependent process. The problem is exacerbated by motion and other temporal features. Trying to foresee which elements will be the most useful for subsequent retrieval is very difficult.

\section{Top-down approaches}

Top-down retrieval systems utilise high-level knowledge of the particular domain to generate appropriate representations. This knowledge can be predefined by expert users, semi-automatically learned or acquired in a completely automatic manner. These categories can differ in the way by which high-level information influences extraction and processing of low-level features. In other words, the system can eliminate unimportant features from the initial feature set, re-processes the features in order to generate new representations or influence the feature generation and analysis at the very stage of extraction and processing.

The majority of existing retrieval systems utilise expert knowledge in a hard-coded manner. The representations are generated in a predetermined way and they are not influenced by high-level information of the analysed media [44].

Techniques such as relevance feedback [40] [50] [54] and incremental machine learning [12] enable intervention of the user in the process of knowledge acquisition. There are many promising examples of semi-automated or semisupervised video retrieval systems that exploit this idea. An approach by Dorado et al. [17] generates the concept lexicon that may consist of words, icons, or any set of symbols that convey the meaning to the user by utilising fuzzy logic and rule mining techniques to approximate human-like reasoning. A nice example of a domain driven semi-automated algorithm for semantic annotation is given by Burghardt [8] where a specific animal face tracker is formed from user labelled examples utilising 
Ada-boost classifier and Kanade-Lucas-Tomasi tracker.

In the work by Bloehdorn et al.[6] a M-OntoMatAnnotizer is designed in order to construct ontologies that include prototypical instances of high-level domain concepts together with a formal specification of corresponding visual descriptors. Thus, it formalizes the interrelationship of high- and low-level multimedia concept descriptions allowing for new kinds of multimedia content analysis and reasoning.

In a recent overview on supervision and statistical learning for semantic multimedia analysis, Naphade [37] outlines that the problem of small sample statistics limits using traditional learning techniques. However, innovations such as labeled and unlabeled learning, active learning and discriminant techniques have made it more feasible to use statistical models for more general video indexing problems. In their earlier work, Naphade et al.[39] defined a factor graph network of probabilistic multimedia objects, multijects, in a probabilistic pattern recognition fashion using hidden Markov and Gaussian mixture models. Another approach that attempts to link a subset of low-level features and words was taken by Barnard et al. in [4] where the joint distribution of image regions and words was learned utilising multi-modal and correspondence extensions to Hofmanns hierarchical clustering/aspect model, a translation model adapted from statistical machine translation and a multi-modal extension to latent dirichlet allocation.

An interesting approach of emergent semantics brings a novel way to create meaning within an analysed collection. Emergent computation, as presented by Staab [48], is based on the idea that appropriate semantic structures might arise purely from the physics of the task environment, rather than from an experts elaborate considerations. Specifically focusing on the image databases, Santini [41] claims that images don't have an intrinsic meaning, but that they are endowed with a meaning by placing them in the context of other images and by the user interaction. Since semantics do shape the representation model, a straightforward implication of this idea is that as well as semantics, video representation depends upon the dynamic of the database and interaction with the user, and cannot be predetermined. The idea of emergent semantics could have far-reaching repercussions in the way that content-based retrieval develops.

There are number of domain specific systems that exploit a unique set of features to form a reliable representation. Such examples include semantic analysis of sports, documentaries, newscasts or soap operas. An algorithm presented by Leonardi et al. in [30, 31] exploits hidden markov models on multimodal data to achieve structural and semantic classification of football videos. On the other hand Bertini and Del Bimbo [5] designed a solution for highlights detection in sports videos using finite state machines that encode the temporal evolution of the analysed highlights.
Bearing in mind that the development and evaluation of such a complex task as multimodal video representation requires a large scale content-management framework, there are number of research projects and initiatives that are addressing this problem.

The target of the aceMedia project [27] is the integration of knowledge and multimedia content technologies, focusing on the benefits of the end user, in the context of a user-centered scenario. In order to simplify the user experience, the aceMedia project focuses its efforts on knowledge discovery and self-adaptability embedded into media content, which will allow it to be self organizing, self annotating, and more readily searched and communicated, by providing tools to automatically analyze content, generate metadata and annotation, and support intelligent content search and retrieval services.

The ICBR (Intelligent Content-based Retrieval) system [10] exploits a unique opportunity to deal with semantic gap issues by integrating a large video database with its semantic description organised in a structured taxonomy. This framework proved to be a unprecedented environment for development of novel representation for semantic video analysis of wildlife documentaries [25, 11, 9].

\section{Conclusions}

As elaborated above, there is a need for more focus on novelties in video representation in order to tackle the problem of semantic gap. In the situation where the type of the index describing a unit of media is defined by descriptors proposed in the MPEG-7 standard and is limited by the set of index terms for which automatic detectors can be realized [47], the prospect of solving the problem of the sematic gap seems rather remote. Introducing a more sophisticated way of representing information embedded in the video media by interacting with the user and analysing more modalities could bring that essential advance to content-based video indexing and retrieval.

Future work will be focused on defining a generic framework for indexing and retrieval of video in order to evaluate different algorithms for video representation and assessing this novel area in a more objective way.

\section{Acknowledgements}

The work reported in this paper has formed part of the activity of the WG3 within IST COST292 action in semantic multimodal analysis of digital media whose funding and support is gratefully acknowledged. For more information please visit www.cost292.org. In addition, the authors would like to acknowledge the support by the EC project aceMedia (FP6-001765) and the ICBR project within the 3C Research programme of convergent technology research for digital media processing and communications. 


\section{References}

[1] B.D. Adams. Where does computational media aesthetics fit? IEEE Multimedia Magazine, spec. ed. Computational Media Aesthetics, April-June 2003.

[2] W. Al-Khatib, Y.F. Day, A. Ghafoor, and P.B. Berra. Semantic modeling and knowledge representation in multimedia databases. IEEE Transactions on Knowledge and Data Engineering, 11(1):64-80, Jan/Feb 1999.

[3] Steve Anderson. Select and combine: The rise of database narratives. Res Magazine, 7(1):52-53, Jan/Feb 2004.

[4] Kobus Barnard, Pinar Duygulu, David Forsyth, Nando de Freitas, David M. Blei, and Michael I. Jordan. Matching words and pictures. J. Mach. Learn. Res., 3:11071135, 2003.

[5] M. Bertini, A. Del Bimbo, and W. Nunziati. Highlights modeling and detection in sports videos. Pattern Analysis and Applications, 2005.

[6] S. Bloehdorn, K. Petridis, C. Saathoff, N. Simou, V. Tzouvaras, Y. Avrithis, S. Handschuh, I. Kompatsiaris, S. Staab, and M. G. Strintzis. Semantic annotation of images and videos for multimedia analysis. In Proceedings of the 2nd European Semantic Web Conference, ESWC 2005, Heraklion, Greece, May 2005.

[7] John S. Boreczky and Lawrence A. Rowe. Comparison of video shot boundary detection techniques. In Storage and Retrieval for Image and Video Databases (SPIE), pages 170-179, 1996.

[8] Tilo Burghardt, Janko Calic, and Barry Thomas. Tracking animals in wildlife videos using face detection. In European Workshop on the Integration of Knowledge, Semantics and Digital Media Technology, October 2004.

[9] Tilo Burghardt, Barry Thomas, Peter J Barham, and Janko Calic. Automated visual recognition of individual african penguins. In Fifth International Penguin Conference, Ushuaia, Tierra del Fuego, Argentina, September 2004.

[10] Janko Calic, Neill Campbell, Majid Mirmehdi, Barry Thomas, Ron Laborde, Sarah Porter, and Nishan Canagarajah. ICBR - multimedia management system for intelligent content based retrieval. In International Conference on Image and Video Retrieval CIVR 2004, pages 601-609. Springer LNCS 3115, July 2004.

[11] Janko Calic and Barry Thomas. Spatial analysis in keyframe extraction using video segmentation. In Workshop on Image Analysis for Multimedia Interactive Services, April 2004.

[12] Gert Cauwenberghs and Tomaso Poggio. Incremental and decremental support vector machine learning. In Proc. of Neural Information Processing Systems (NIPS) 2000, Denver, USA.

[13] Glorianna Davenport, Thomas Aguirre Smith, and Natalio Pincever. Cinematic primitives for multimedia. IEEE Comput. Graph. Appl., 11(4):67-74, 1991.

[14] Marc Davis. Media streams: representing video for retrieval and repurposing. In MULTIMEDIA '94: Proceedings of the second ACM international conference on Multimedia, pages 478-479, New York, NY, USA, 1994. ACM Press.
[15] Marc Davis. Media streams: representing video for retrieval and repurposing. $\mathrm{PhD}$ thesis, Cambridge, MA, USA, 1995.

[16] Nevenka Dimitrova. Context and memory in multimedia content analysis. IEEE MultiMedia, 11(3):7-11, 2004.

[17] A. Dorado, J. Calic, and E. Izquierdo. A rule-based video annotation system. Circuits and Systems for Video Technology, IEEE Transactions on, 14(5):622-633, May 2004.

[18] Chitra Dorai and Svetha Venkatesh. Media computing: computational media aesthetics. The Kluwer international series in video computing. Kluwer Academic Publishers, Boston; London, 2002.

[19] Venkatesh S. Dorai, C. Bridging the semantic gap with computational media aesthetics. Multimedia, IEEE, 10:15-17, 2003.

[20] Sergei Eisenstein and Jay Leyda. Film Form Essays in film theory. Dennis Dobson Ltd, [S.1.], 1949. edited and translated by Jay Leyda.

[21] M. Flickner, H. Sawhney, W. Niblack, J. Ashley, Q. Huang, B. Dom, M. Gorkani, J. Hafner, D. Lee, D. Petkovic, D. Steele, and P. Yanker. Query by image and video content: The qbic system. IEEE Computer, 28(9):23-32, 1995.

[22] U. Gargi, R. Kasturi, and S. H. Strayer. Performance characterization of video-shot-change detection methods. Circuits and Systems for Video Technology, IEEE Transactions on, 10(1):1, 2000.

[23] Louis D. Giannetti. Understanding movies. Prentice Hall; London: Prentice-Hall International (UK), Upper Saddle River, N.J., 9th ed. edition, 2002.

[24] A. Hanjalic and L. Q. Xu. Affective video content representation and modeling. IEEE Transactions on Multimedia, 7(1):143-154, 2005.

[25] Sion Hannuna, Neill Campbell, and David Gibson. Segmenting quadruped gait patterns from wildlife video. In The IEE International Conference on Visual Information Engineering: Convergence in Graphics and Vision., pages 235-243. Institution of Electrical Engineers, April 2005.

[26] S. Kiranyaz, K. Caglar, E. Guldogan, and M. Gabbouj. Muvis: A content-based multimedia indexing and retrieval framework. In Proc. of the Third International Workshop on Content-Based Multimedia Indexing, CBMI 2003, pages 405-412, Rennes, France, Sept. 2003.

[27] I. Kompatsiaris, Y. Avrithis, P. Hobson, T. May, and J. Tromp. Achieving Integration of Knowledge and Content Technologies: The AceMedia Project. In Proc. European Workshop on the Integration of Knowledge, Semantics and Digital Media Technology, Royal Statistical Society, London, UK, Nov. 2004.

[28] Irena Koprinska and Sergio Carrato. Temporal video segmentation: A survey. Signal Processing: Image Communication, 16(5):477, 2001.

[29] Lev Kuleshov and Ronald Levaco. Kuleshov on film: writings by Lev Kuleshov. University of California Press, Berkeley; London, 1974.

[30] R. Leonardi and P. Migliorati. Semantic indexing of multimedia documents. Multimedia, IEEE, 9(2):44, 2002. 
[31] R. Leonardi, P. Migliorati, and M. Prandini. Semantic indexing of soccer audio-visual sequences: a multimodal approach based on controlled Markov chains. Circuits and Systems for Video Technology, IEEE Transactions on, 14(5):634, 2004.

[32] Dongge Li, Nevenka Dimitrova, Mingkun Li, and Ishwar K. Sethi. Multimedia content processing through cross-modal association. In MULTIMEDIA '03: Proceedings of the eleventh ACM international conference on Multimedia, pages 604-611, New York, NY, USA, 2003. ACM Press.

[33] Lev Manovich. The language of new media. Leonardo. MIT Press, Cambridge, Mass.; London, 2001.

[34] Christian Metz. [Essais sur la signification au cinéma.] Film language. A semiotics of the cinema. Translated by Michael Taylor. New York: Oxford University Press, 1974.

[35] V. Mezaris, H. Doulaverakis, S. Herrmann, B. Lehane, N. OConnor, I. Kompatsiaris, and M. G. Strintzis. The SCHEMA Reference System: An Extensible Modular System for Content-based Information Retrieval. In Proc. Workshop on Image Analysis For Multimedia Interactive Services (WIAMIS), Montreux, Switzerland, April. 2005.

[36] F. Nack and A. Parkes. Toward the automated editing of theme-oriented video sequences. Applied Artificial Intelligence, 11(4):331-366, 1997.

[37] Milind R. Naphade. On supervision and statistical learning for semantic multimedia analysis. Journal of Visual Communication and Image Representation, 15(3):348369, 2004.

[38] Laurence Nigay and Joëlle Coutaz. A design space for multimodal systems: concurrent processing and data fusion. In CHI '93: Proceedings of the SIGCHI conference on Human factors in computing systems, pages 172-178, New York, NY, USA, 1993. ACM Press.

[39] M. Ramesh Naphade, I. V. Kozintsev, and T. S. Huang. Factor graph framework for semantic video indexing. Circuits and Systems for Video Technology, IEEE Transactions on, 12(1):40, 2002.

[40] J.J. Rocchio, Jr. The SMART Retrieval System: Experiments in Automatic Document Processing, chapter Relevance Feedback in Information Retrieval, pages 313-323. Prentice-Hall, 1971.

[41] S. Santini, A. Gupta, and R. Jain. Emergent semantics through interaction in image databases. Knowledge and Data Engineering, IEEE Transactions on, 13(3):337, 2001.

[42] C. Saraceno and R. Leonardi. Indexing audiovisual databases through joint audio and video processing. IJIST, 9(5):320-331, 1999.

[43] Ferdinand de Saussure. Course in general linguistics. Duckworth, London, 1983.

[44] Arnold W. M. Smeulders, Marcel Worring, Simone Santini, Amarnath Gupta, and Ramesh Jain. Content-based image retrieval at the end of the early years. IEEE Trans. Pattern Anal. Mach. Intell., 22(12):1349-1380, 2000.
[45] J.R. Smith and S.F. Chang. Tools and techniques for color image retrieval. In Storage and Retrieval for Image and Video Databases (SPIE), pages 426-437, 1996.

[46] J.R. Smith and S.F. Chang. Image and video search engine for the world wide web. In Storage and Retrieval for Image and Video Databases (SPIE), pages 84-95, 1997.

[47] C.G.M. Snoek and M. Worring. Multimodal video indexing: A review of the state-of-the-art. Multimedia Tools and Applications, 25(1):5-35, 2005.

[48] S. Staab. Emergent semantics. IEEE Intelligent Systems, 17(1):78-81, 2002.

[49] J. Vendrig and M. Worring. Systematic evaluation of logical story unit segmentation. IEEE Transactions on Multimedia, 4(4):492-499, 2002.

[50] M. E. J. Wood, N. W. Campbell, and B. T. Thomas. Iterative refinement by relevance feedback in content-based digi tal image retrieval. In ACM Multimedia 98, pages 13-20. ACM, September 1998.

[51] Wang Yao, Liu Zhu, and Huang Jin-Cheng. Multimedia content analysis-using both audio and visual clues. Signal Processing Magazine, IEEE, 17(6):12, 2000.

[52] A. Yoshitaka and T. Ichikawa. A survey on content-based retrieval for multimedia databases. IEEE Transactions on Knowledge and Data Engineering, 11(1):81-93, Jan/Feb 1999.

[53] HongJiang Zhang, Atreyi Kankanhalli, and Stephen W. Smoliar. Automatic partitioning of full-motion video. Multimedia Syst., 1(1):10-28, 1993.

[54] Xiang Sean Zhou and Thomas S. Huang. Relevance feedback in image retrieval: A comprehensive review. Multimedia Syst., 8(6):536-544, 2003. 